\title{
Polarisabilities of the proton and neutron from Compton scattering
}

\author{
Judith A. McGovern* \\ University of Manchester \\ E-mail: judith.mcgovern@manchester.ac.uk
}

\author{
Harald W. Grießhammer \\ George Washington University \\ E-mail: hgrieegwu.edu
}

\section{Daniel R. Phillips}

Ohio University

E-mail: phillips@phy.ohiou.edu

We have recently completed a high-precision extraction of the proton spin-independent polarisabilities from the world database of low-energy Compton scattering experiments, within the framework of chiral effective field theory ( $\chi \mathrm{EFT})$ with pions, nucleons, and the Delta(1232) as explicit degrees of freedom. Our Baldin-sum-rule-constrained results are, in units of $10^{-4} \mathrm{fm}^{3}$,

$$
\begin{aligned}
& \alpha_{E 1}^{\mathrm{p}}=10.65 \pm 0.35 \text { (stat) } \pm 0.2 \text { (Baldin) } \pm 0.3 \text { (theory) }, \\
& \beta_{M 1}^{\mathrm{p}}=3.15 \mp 0.35 \text { (stat) } \pm 0.2 \text { (Baldin) } \mp 0.3 \text { (theory). }
\end{aligned}
$$

These were obtained in the heavy-baryon formulation, but almost identical results have been obtained in a covariant calculation.

With the publication this year by Myers et al. of the results of the deuteron Compton scattering experiment using the Tagged-Photon Facility at the MAX IV Laboratory in Lund, Sweden, the world database has doubled in size, allowing the extraction of the isoscalar polarisabilities with unprecedented accuracy, and combined with the proton results we obtain

$$
\begin{aligned}
& \alpha_{E 1}^{\mathrm{n}}=11.65 \pm 1.25 \text { (stat) } \pm 0.2 \text { (Baldin) } \pm 0.8 \text { (theory) }, \\
& \beta_{M 1}^{\mathrm{n}}=3.55 \mp 1.25(\text { stat }) \pm 0.2 \text { (Baldin) } \mp 0.8 \text { (theory). }
\end{aligned}
$$

A new generation of experiments with polarised beams have been performed at the Mainz Microtron, with the first results published this year by Martel et al. These experiments are sensitive to the spin polarisabilities, and we will discuss the predictions of $\chi \mathrm{EFT}$ for the relevant cross sections and asymmetries.

The 8th International Workshop on Chiral Dynamics

29 June 2015 - 03 July 2015

Pisa, Italy

${ }^{*}$ Speaker. 


\section{Introduction}

Chiral dynamics in the baryonic sector is typically thought of as the study of the interactions of pions and nucleons. However the dictates of electromagnetic gauge invariance mean that chiral symmetry also strongly constrains the interactions of both with photons, and so Compton scattering from the nucleon is as fundamental a probe of chiral dynamics as pion-nucleon or nucleon-nucleon scattering. The lowest-order term in the Compton scattering amplitude (the long-wavelength limit) is the Thomson term which is reproduced by $\chi \mathrm{PT}$ but which, depending as it does only on the nucleon charge and mass, is independent of chiral dynamics. However, at shorter wavelengths the probing proton starts to be sensitive to the structure of the target. At NLO in heavy baryon chiral perturbation theory $(\mathrm{HB} \chi \mathrm{PT})$ the dominant new contribution comes from a single pion loop with photons coupling to the pion or to the $\pi \mathrm{N}$ vertex (see Fig. 1), and hence a prediction can be made for these structure effects. This includes, but is not limited to, the numbers known as the polarisabilities of the nucleon; the latter are the first terms in an expansion in powers of the photon energy of the lowest $(l=1)$ scattering multipoles.

The application of chiral dynamics to Compton scattering dates back to the dawn of baryon $\chi \mathrm{PT}$, and most famously the lowest-order predictions for the electric and magnetic polarisability of the proton in $\mathrm{HB} \chi \mathrm{PT}, \alpha_{E 1}^{\mathrm{p}}=12.5 \times 10^{-4} \mathrm{fm}^{3}$ and $\beta_{M 1}^{\mathrm{p}}=1.2 \times 10^{-4} \mathrm{fm}^{3}$ [1] were in very good agreement with the experimental determinations of the time. Higher-order calculations [2, 3], and calculations of spin polarisabilities $[4,5,6,7,8]$ followed. At fourth order the scalar polarisabilities obtain contributions from low-energy constants, which can only be obtained by fitting Compton scattering at finite energies. The first studies to systematically compare the full predicted cross section to a compendium of experimental data for the proton and deuteron were published by some of the current authors, first without $[9,10,11]$ and then with [12] the inclusion of the Delta resonance as an explicit degree of freedom. These studies obtained an excellent fit to data at low energies (though without the Delta, a cut on momentum transfer was required), and clearly demonstrated the pion-production cusp. Both of them in addition found values of $\beta_{M 1}^{\mathrm{p}}$ around $3.4 \times$ $10^{-4} \mathrm{fm}^{-3}$, substantially larger than the accepted values at this time, albeit still with significant uncertainties.

In recent years there has been an upsurge in interest in the polarisabilities of the nucleon, both scalar and spin, with a number of new experiments planned, running or completed $[13,14,15,16$, $17,18,19]$. In addition the magnetic polarisability $\beta_{M 1}^{\mathrm{p}}$ has been shown to be a crucial input in the determination of the two-photon-exchange contribution to the Lamb shift in muonic hydrogen [20, $21,22,23$ ], and the isovector $\beta_{M 1}^{\mathrm{p}}-\beta_{M 1}^{\mathrm{n}}$ has been connected to the nucleon electromagnetic mass difference $[25,24,26,27,28]$. The calculation of nucleon polarisabilities is also an aim of lattice QCD, and several groups now have published results [29, 30, 31, 32, 33, 34, 35, 36, 37, 38], albeit almost all at large pion masses. In this contribution we report on the results of high-precision EFT fits of scalar polarisabilities to current data, and on prospects for the determination of spin polarisabilities. For more details the reader is referred to our review [39], and to the papers detailing the extractions of the scalar polarisability from the proton [40] and neutron [18]. For related work see the contributions of H. Grießhammer, E. Downie, J. Annand, G. Feldman, H. Gao and B. Demissie in these proceedings.

For completeness we reproduce the low-energy non-relativistic effective Hamiltonian that in- 
dicates how polarisabilities affect the response of the nucleon to external electric and magnetic fields [41]:

$H_{\mathrm{eff}}=-\frac{1}{2} 4 \pi\left(\alpha_{E 1} \vec{E}^{2}+\beta_{M 1} \vec{H}^{2}+\gamma_{E 1 E 1} \vec{\sigma} \cdot \vec{E} \times \vec{E}+\gamma_{M 1 M 1} \vec{\sigma} \cdot \vec{H} \times \vec{H}-2 \gamma_{M 1 E 2} E_{i j} \sigma_{i} H_{j}+2 \gamma_{E 1 M 2} H_{i j} \sigma_{i} E_{j}\right)$,

where dots mean a time derivative and $X_{i j}=\frac{1}{2}\left(\nabla_{i} X_{j}+\nabla_{j} X_{i}\right)$. The scalar polarisabilities will be given throughout in units of $10^{-4} \mathrm{fm}^{3}$, and the spin polarisabilities in units of $10^{-4} \mathrm{fm}^{4}$.

\section{Chiral EFT and $\delta$ power counting}

Here we discuss the relevant terms in the Lagrangians for the construction of the Compton scattering amplitude to fourth order in $\mathrm{HB} \chi \mathrm{PT}$. The full Lagrangian needs to be written in terms of building blocks with appropriate chiral properties, and hence every term can give rise to interactions with multiple pions. The usual notation is compact but far from transparent. Below we retain only the relevant structures for our purposes:

$$
\begin{aligned}
\mathscr{L}_{\pi}^{(2)}= & \frac{1}{2} \partial_{\mu} \phi \cdot \partial^{\mu} \phi+e A_{\mu} \varepsilon_{3 i j} \phi_{i} \partial^{\mu} \phi_{j}+\frac{1}{2} e^{2} A_{\mu} A^{\mu}\left(\phi_{1}^{2}+\phi_{2}^{2}\right)-\frac{1}{2} m_{\pi}^{2} \phi^{2}+\ldots \\
\mathscr{L}_{\pi \mathrm{N}}^{(1)}= & \psi^{\dagger}\left(\mathrm{i} v \cdot D+g_{A} u \cdot S\right) \psi \\
\mathscr{L}_{\pi \mathrm{N}}^{(2)}= & \psi^{\dagger}\left\{\frac{1}{2 M_{\mathrm{N}}}\left((v \cdot D)^{2}-D^{2}-\mathrm{i} g\{S \cdot D, v \cdot u\}\right)+4 c_{1} m_{\pi}^{2}\left(1-\frac{1}{2 f_{\pi}^{2}} \phi^{2}\right)\right. \\
& \left.+\left(c_{2}-\frac{g_{A}^{2}}{8 M_{\mathrm{N}}}\right)(v \cdot u)^{2}+c_{3} u \cdot u-\frac{\mathrm{i}}{4 M_{\mathrm{N}}}\left[S^{\mu}, S^{\nu}\right] e F_{\mu v}\left(\left(1+\kappa^{(\mathrm{s})}\right)+\left(1+\kappa^{(\mathrm{v})}\right) \tau_{3}\right)\right\} \psi+\ldots \\
\mathscr{L}_{\pi \mathrm{N}}^{(4)}= & 2 \pi e^{2} \psi^{\dagger}\left\{\frac{1}{2}\left(\delta \beta^{(\mathrm{s})}+\delta \beta^{(\mathrm{v})} \tau_{3}\right) g_{\mu v}\right. \\
& \left.-\left(\left(\delta \alpha^{(\mathrm{s})}+\delta \beta^{(\mathrm{s})}\right)+\left(\delta \alpha^{(\mathrm{v})}+\delta \beta^{(\mathrm{v})}\right) \tau_{3}\right) v_{\mu} v_{v}\right\} F^{\mu \rho} F_{\rho}^{v} \psi+\ldots
\end{aligned}
$$

where $\psi$ is the nucleon field, $\phi_{a}$ are the pion fields, $F^{\mu v}$ is the electromagnetic field tensor and $D^{\mu} \equiv \partial^{\mu}-\mathrm{i} e Q A^{\mu}$ is the gauged derivative; $v^{\mu}=g^{0 \mu}$ and $S^{\mu}=(0, \vec{\sigma} / 2)$ in the rest frame of the nucleon. The object $u_{\mu}$ is given by

$$
u_{\mu}=-\frac{1}{f_{\pi}}\left(\tau_{a} \partial_{\mu} \phi_{a}+e \varepsilon^{a 3 b} \tau_{a} \phi_{b} A_{\mu}+\ldots\right)
$$

The $\Delta(1232)$ resonance has long been recognised as hugely important in the physics of nucleons. In $\chi \mathrm{PT}$ it is not explicitly present, but its influence is felt through LECs such as $c_{2,3}$ and $\delta \beta_{M 1}^{\mathrm{p}}$. But, as the radius of convergence of an EFT is set by the scale of the lowest degree of freedom which has not been included, the $\Delta(1232)$ can be expected to severely restrict the applicability of $\chi \mathrm{PT}$ at least in those processes in which it contributes, with the convergence governed by the scale $\Delta_{M} \equiv M_{\Delta}-M_{\mathrm{N}}$. And any glance at Compton data above $200 \mathrm{MeV}$, as in Fig. 3, confirms that this is such a process. Thus in order to make best use of current data, the inclusion of the Delta as an explicit degree of freedom is mandatory [42, 43, 44].

The relevant terms in the heavy-baryon Lagrangian are the following: 


$$
\begin{aligned}
& \mathscr{L}_{\Delta}^{(1)}=\left(\Delta_{v}^{i}\right)^{\dagger}(-\mathrm{i} v \cdot D+\Delta) \Delta^{i v} \\
& \mathscr{L}_{\pi \mathrm{N} \Delta}^{(1)}=-\frac{g_{\pi \mathrm{N} \Delta}}{f_{\pi}}\left(\psi^{\dagger} \partial^{v} \phi^{i} \Delta_{v}^{i}+\left(\Delta_{v}^{i}\right)^{\dagger} \partial^{v} \phi^{i} \psi+\ldots\right) \\
& \mathscr{L}_{\gamma \mathrm{N} \Delta}^{(2)}=\frac{-\mathrm{i} e b_{1}}{M_{\mathrm{N}}}\left(\psi^{\dagger} S_{\rho} F^{\mu \rho} \Delta_{\mu}^{3}-\left(\Delta_{\mu}^{3}\right)^{\dagger} S_{\rho} F^{\mu \rho} \psi\right)
\end{aligned}
$$

where $\Delta_{v}^{i}$ is the heavy-baryon reduction of an $I=\frac{3}{2}, S=\frac{3}{2}$ Rarita-Schwinger field $\Psi_{v}^{i}$, with $i$ and $\mu$ the indices on the (iso)spin-1 vector coupled to the (iso)spin- $\frac{1}{2}$ spinor. We also show the alternative form of $\mathscr{L}_{\gamma \mathrm{N} \Delta}^{(2+3)}$ used in the $\delta$-expansion, see later (here $\psi$ is the nucleon Dirac spinor and other notation is that of Ref. [45]):

$$
\mathscr{L}_{\gamma \mathrm{N} \Delta}^{\text {cov }}=\frac{3 e}{2 M_{\mathrm{N}}\left(M_{\mathrm{N}}+M_{\Delta}\right)}\left(\bar{\psi}\left(\mathrm{i} g_{M} \tilde{F}^{\mu v}-g_{E} \gamma_{5} F^{\mu v}\right) \partial_{\mu} \Psi_{v}^{3}-\bar{\Psi}_{v}^{3} \overleftarrow{\partial}_{\mu}\left(\mathrm{i} g_{M} \tilde{F}^{\mu v}-g_{E} \gamma_{5} F^{\mu v}\right) \psi\right)
$$

The leading (magnetic) term in the heavy-baryon reduction of this Lagrangian is equivalent to the one above with the identification $g_{M}=b_{1}\left(1+M_{\Delta} / M_{\mathrm{N}}\right) / 3$ (though it is important to note that if the full vertex is used, there are substantial sub-leading terms) but there is also a sub-leading electric coupling $g_{E}$. The ratio of these two couplings (at the $\Delta(1232)$ pole) can be obtained from the E2/M1 ratio to be $-0.34[46,47]$. Being third order, the electric contribution to the amplitudes is suppressed by a power of $\omega / M_{\mathrm{N}}$ relative to the magnetic one.

It has been argued that since $\Delta_{M}$ is not much more than twice the pion mass, the two should be counted similarly (the so called "small-scale" or " $\varepsilon$ " expansion). However, important as the $\Delta(1232)$ is above the photoproduction threshold, its influence on the cross section diminishes rapidly as the energy is reduced. Arguably, counting $\Delta_{M}$ and $m_{\pi}$ as the same scale gives undue prominence to the $\Delta(1232)$ in the region where static polarisabilities are important. An alternative counting was proposed by Pascalutsa and Phillips [45] in which $m_{\pi} / \Delta_{M}$ and $\Delta_{M} / \Lambda_{\chi}$ are counted as proportional to the same expansion parameter $\delta$ (the so-called " $\delta$-expansion"). In this counting, for low energies, the $\Delta$-less theory is an expansion in powers of $\delta^{2}$, and the first contributions from the $\Delta$ (the pole diagrams and the $\pi \Delta$ loops) intercalate between the third and fourth orders of $\chi$ PT. The main advantage of this expansion, however, is that it allows for two separate energy regions, $\omega \sim m_{\pi}$ and $\omega \sim \Delta_{M}$. In the latter regime there is no suppression of $\pi \mathrm{N}$ loop contributions to the $\Delta$ propagator. These must thus be resummed, and the $\Delta$ becomes an unstable particle with a width $\Gamma$, the new propagator going as $\left(\not p-M_{\Delta}+\mathrm{i} \Gamma\left(p^{2}\right) / 2\right)^{-1}$. For $\omega \sim \Delta_{M}$, the one- $\Delta$ reducible diagram dominates (that is, the direct $\Delta$-pole diagram), with all other contributions being sub-leading. The first corrections to the $\Delta$-pole diagram come from pion loops at the $\gamma \mathrm{N} \Delta$ vertex, which lead to a running of the effective magnetic and electric couplings, and more importantly give them imaginary parts above the photoproduction threshold which enable Watson's theorem to be satisfied. These were first calculated in Ref. [46] in the context of photoproduction. In what follows we will use the HB Lagrangian of Eqs (2.1-2.8) for everything except the Delta-pole graph. For that we will use the covariant form (2.9) with running couplings; this will improve the description of the kinematics in the resonance region. 


\begin{tabular}{|c|c|c|c|}
\hline & contribution with typical size & $\omega \sim m_{\pi}$ & $\omega \sim \Delta$ \\
\hline (i) & & $e^{2} \delta^{0}(\mathrm{LO})$ & $e^{2} \delta^{0}$ \\
\hline (ii) & (c) & $e^{2} \delta^{2}$ & $e^{2} \delta^{1}$ \\
\hline (iii) & & $e^{2} \delta^{4}$ & $e^{2} \delta^{2}$ \\
\hline
\end{tabular}

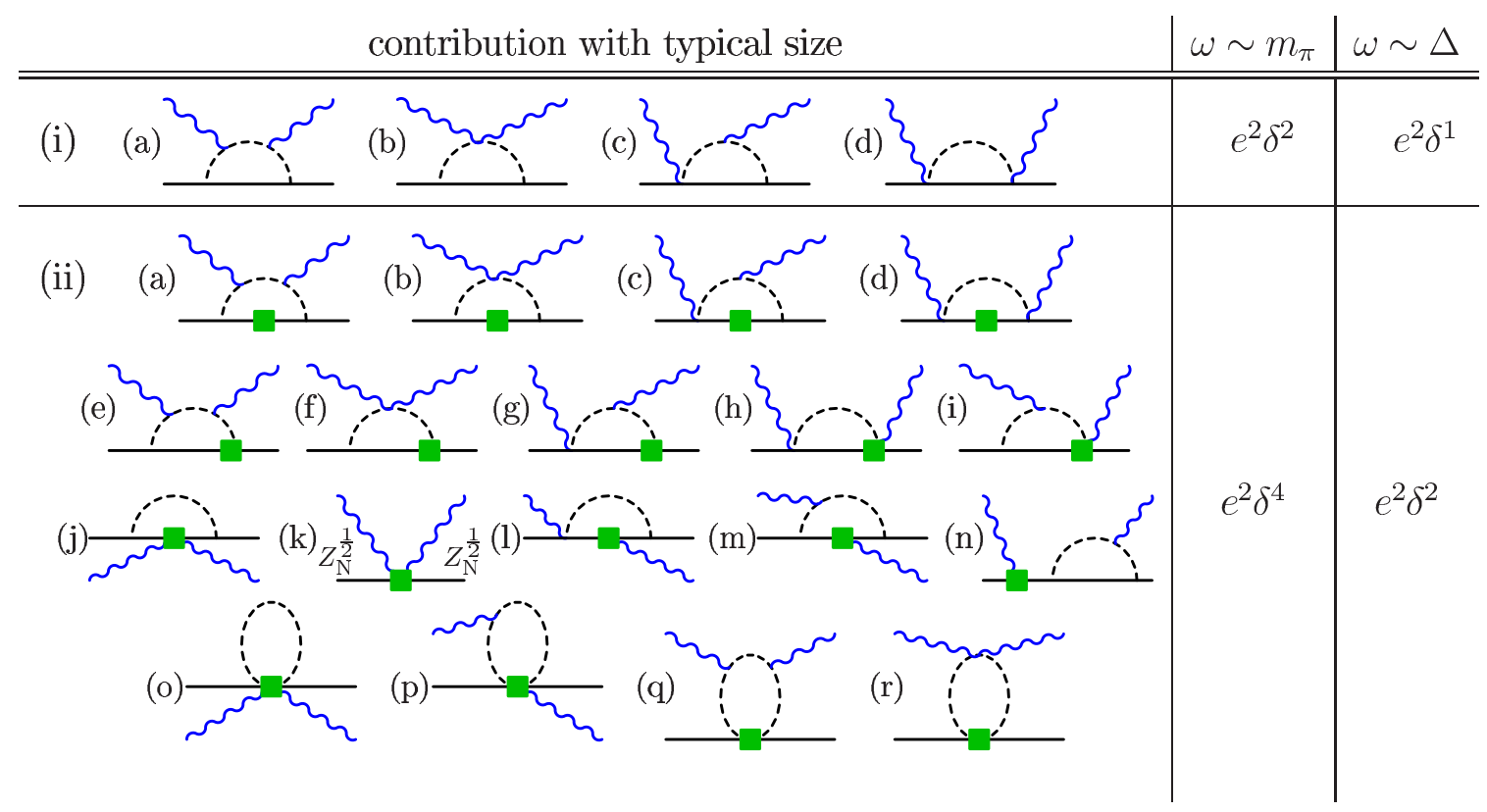

Figure 1: Tree and pion-nucleon loop diagrams that contribute to Compton scattering in the $\varepsilon \cdot v=0$ gauge, ordered by the typical size of their contributions in the two regimes $\omega \sim m_{\pi} \sim \delta^{2}$ and $\omega \sim \Delta_{M} \sim \delta$, respectively. The leading-order contribution in a particular regime is indicated by (LO). The vertices are from: $\mathscr{L}_{\pi \mathrm{N}}^{(1)}$ (no symbol), $\mathscr{L}_{\pi \mathrm{N}}^{(2)}$ (square), $\mathscr{L}_{\pi \mathrm{N}}^{(3)}$ (triangle), $\mathscr{L}_{\pi \mathrm{N}}^{(4)}$ (diamond), $\mathscr{L}_{\pi \pi}^{(4)}$ (disc). Permuted and crossed diagrams not shown. Figure reproduced from ref. [40]

\section{Compton Scattering from the proton}

The diagrams used to calculate the Compton scattering amplitudes to $\mathrm{N}^{3} \mathrm{LO}$ at low energies and to NLO in the Delta region are given in figures 1 and 2. From these we construct the $\gamma p$ scattering cross section which depends, effectively, on only three unknowns: the $\gamma \mathrm{N} \Delta$ magnetic coupling constant $g_{M}$, and the 4th-order LECs $\delta \alpha$ and $\delta \beta$ which contribute to the proton scalar polarisabilities (see Eq. (2.4); however instead of quoting these, which in any case are scale-dependent, we always quote the full values of the polarisabilities.) Our strategy is to iteratively fit $g_{M}$ to data around the Delta peak, where the cross section is extremely sensitive to it, while fitting $\alpha_{E 1}^{\mathrm{p}}$ and 


\begin{tabular}{|c|c|c|c|}
\hline & contribution with typical size & $\omega \sim m_{\pi}$ & $\omega \sim \Delta$ \\
\hline (i) & & $e^{2} \delta^{3}$ & $e^{2} \delta^{-1}(\mathrm{LO})$ \\
\hline (ii) (a) & & $e^{2} \delta^{3}$ & $e^{2} \delta^{1}$ \\
\hline
\end{tabular}

\begin{tabular}{|c|c|c|}
\hline contribution with typical size & $\omega \sim m_{\pi}$ & $\omega \sim \Delta$ \\
\hline (i) & $e^{2} \delta^{2}$ & $e^{2} \delta^{1}$ \\
\hline (ii) (a) & $e^{2} \delta^{4}$ & $e^{2} \delta^{2}$ \\
\hline (iii) & $e^{2} \delta^{6}$ & $e^{2} \delta^{3}$ \\
\hline
\end{tabular}

Figure 2: $\Delta(1232)$ pole and $\Delta \pi$ loop diagrams. Notation as in Fig. 1, also double line: $\Delta(1232)$; shaded blob: $\pi \mathrm{N} \Delta$ couplings including vertex corrections. $\gamma \mathrm{N} \Delta$ vertices from $\mathscr{L}_{\gamma \mathrm{N} \Delta}^{\text {cov }}$, Eq. (2.9), at $\mathscr{O}\left(e \delta^{2}\right)$ proportional to $g_{M}$ (square), and at $\mathscr{O}\left(e \delta^{3}\right)$ proportional to $g_{E}$ (triangle). Permuted and crossed diagrams not shown. Figure reproduced from ref. [40]

$\beta_{M 1}^{\mathrm{p}}$ to the low energy data, taken to be below $170 \mathrm{MeV}$. In practice we find that both the Delta pole graph and 4th-order pion-nucleon loops give large contributions to the spin polarisability $\gamma_{M 1 M 1}$, leading to a predicted value substantially higher than that suggested from other sources, and to a poor fit to data. Including $\gamma_{M 1 M 1}$ as a fit parameter, however, gives a huge improvement in the fit and a much more natural value of that polarisability. Our results for $\alpha_{E 1}^{\mathrm{p}}$ and $\beta_{M 1}^{\mathrm{p}}$ are compatible with the Baldin sum rule $\alpha_{E 1}^{\mathrm{p}}+\beta_{M 1}^{\mathrm{p}}=13.8 \pm 0.4$, so we reduce our statistical errors by imposing that constraint. The final result is $\alpha_{E 1}^{\mathrm{p}}-\beta_{M 1}^{\mathrm{p}}=7.5 \pm 0.7$ (stat) \pm 0.6 (theory) hence

$$
\begin{aligned}
& \alpha_{E 1}^{\mathrm{p}}=10.65 \pm 0.35 \text { (stat) } \pm 0.2 \text { (Baldin) } \pm 0.3 \text { (theory), } \\
& \beta_{M 1}^{\mathrm{p}}=3.15 \mp 0.35 \text { (stat) } \pm 0.2 \text { (Baldin) } \mp 0.3 \text { (theory), }
\end{aligned}
$$

with $\gamma_{M 1 M 1}=2.2 \pm 0.5$ (stat) and $g_{M}=2.78 \pm 0.02$ (stat), with a $\chi^{2}$ of 113.2 for 135 d.o.f. Units throughout are $10^{-4} \mathrm{fm}^{-3}$ for scalar polarisabilities, and $10^{-4} \mathrm{fm}^{-4}$ for spin polarisabilities.

The theory error was estimated from order-by-order convergence of $\alpha_{E 1}-\beta_{M 1}$, from its LO value to the NNLO fit given above. (More details on the convergence are given in the contribution of H. Grießhammer in these proceedings [49].)

The data set from which we obtained this result was first tested for statistical consistency. Above $200 \mathrm{MeV}$, it is well known that the LEGS [48] and SAL [50] data are not consistent with that from Mainz [51], and as the latter is much more copious we chose to use it. At lower energies we found that the SAL data is entirely compatible with world data up to the cusp but not beyond; we also discarded a couple of very old data sets, and a couple of individual points. While necessary 
for a good $\chi^{2}$, we checked that none of this had a significant effect on the final extracted values. See Refs $[39,40]$ for more details. The predictions of the chiral theory with these fit parameters are shown together with world data in Fig. 3.

At this point we should note that Compton scattering on the proton has also been investigated in the covariant, rather than heavy baryon, formulation, working to NNLO, by Lensky and Pascalutsa [53, 54]. A comparison of the predictions of the two formulations was given in Ref. [55]. If (though it is not strictly mandated at this order) $\alpha_{E 1}^{\mathrm{p}}$ and $\beta_{M 1}^{\mathrm{p}}$ are regarded as fit parameters, the results are in almost exact agreement with those of Eq. (3.1) [56].

\section{Compton Scattering from the deuteron}

In the absence of free neutron targets, the best prospects for extracting the neutron polarisabilities currently come from Compton scattering from the deuteron. Chiral EFT can be extended to the two nucleon sector by supplementing the Lagrangian with NN contact interactions, which encode the short-range, non-chiral physics that binds the deuteron, while pion exchange governs the long-distance tail of the wave function; after fitting to deuteron and NN scattering data there are no further free parameters beyond the LECs for the isoscalar polarisabilities. The two-nucleon Compton scattering kernel can be calculated, as shown in Fig. 4; it has one-body terms which consist of the diagrams of Fig. 1, 2 with a spectator nucleon, and two-body diagrams where the photons couple to exchanged pions or $\pi \mathrm{N}$ vertices. At chiral energies $\omega \sim m_{\pi}$ any graph in which the two nucleons interact between photon absorption and re-emission are higher order, but at low energies such graphs are enhanced, and indeed as $\omega \rightarrow 0$ they are required to ensure the correct Thomson limit. Since the world deuteron data starts as low as $49 \mathrm{MeV}$, an improved description of the data is obtained with the inclusion of rescattering, as demonstrated by Hildebrandt et al. in Ref. [57]. At the order to which we work, there are no two-body graphs with Deltas.

In Ref. [39] we fitted $\alpha_{E 1}^{(\mathrm{s})}$ and $\beta_{M 1}^{(\mathrm{s})}$ to the pre-2014 world deuteron data, then in Ref. [18] the fit was updated to include the new MAX-lab data which almost doubled the size of the database. A couple of points should be noted; the one-body diagrams were implemented to NNLO, rather than $\mathrm{N}^{3} \mathrm{LO}$ as in the proton case; and for simplicity the heavy-baryon form of the $\gamma \mathrm{N} \Delta$ Lagrangian was used. The main consequence of this lower-order fit is a larger theory error than for the proton; however the statistical error still dominates. The isoscalar Baldin sum rule of $\alpha_{E 1}^{(\mathrm{s})}+\beta_{M 1}^{(\mathrm{s})}=14.5 \pm$ 0.4 , [58] was used as a constraint. We obtained $\alpha_{E 1}^{(\mathrm{s})}-\beta_{M 1}^{(\mathrm{s})}=7.8 \pm 1.2$ (stat) \pm 0.8 (th), with a $\chi^{2}$ of 45.2 for 44 degrees of freedom.

This was then combined with the proton value to extract numbers for the neutron:

$$
\begin{aligned}
& \alpha_{E 1}^{\mathrm{n}}=11.65 \pm 1.25(\mathrm{stat}) \pm 0.2 \text { (Baldin) } \pm 0.8 \text { (theory), } \\
& \beta_{M 1}^{\mathrm{n}}=3.55 \mp 1.25(\text { stat }) \pm 0.2 \text { (Baldin) } \mp 0.8 \text { (theory), }
\end{aligned}
$$

The world data and chiral EFT cross sections are shown in Fig. 5.

\section{Beyond the cross section}

Until now most Compton scattering data by far are unpolarised cross sections; the only exception at the time of our proton fit was some beam-asymmetry data from LEGS. This is changing with 

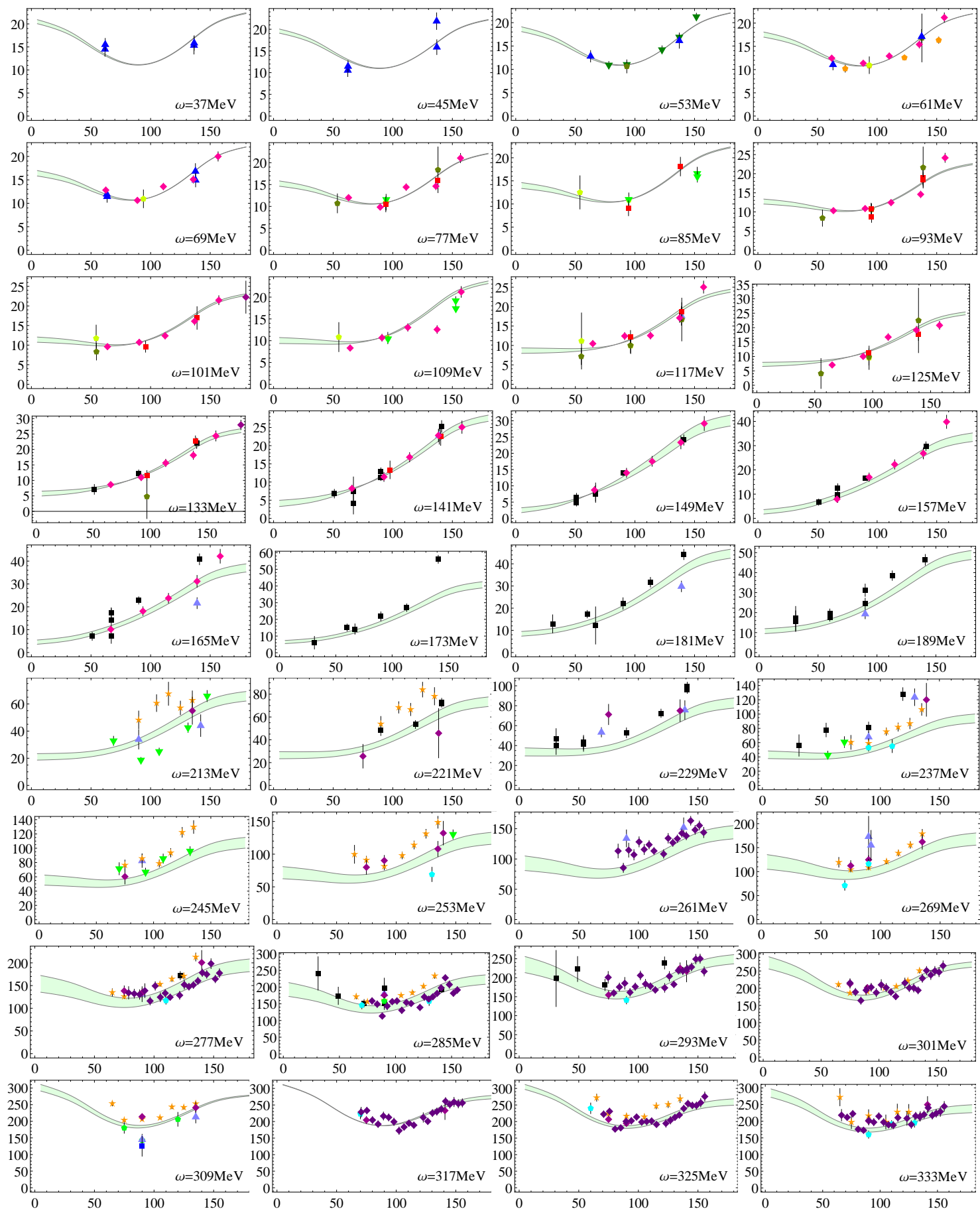

Figure 3: World proton Compton scattering data and chiral EFT predictions. The labelled photon laboratory energy is the central value of $8 \mathrm{MeV}$ bins, and the shaded bands span the same range (variation due to the errors on the extracted polarisabilities in small in comparison). The symbols are explained in Table 3.1 of Ref. [39], but notable are purple diamonds for Mainz (mostly Refs [51, 52]), black squares for SAL [50] and yellow stars for LEGS [48]. 
(a):

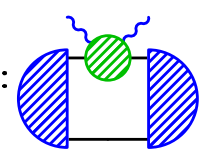

(b):

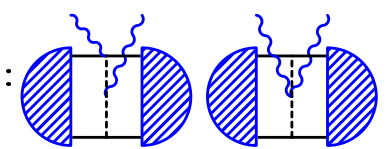

(c):

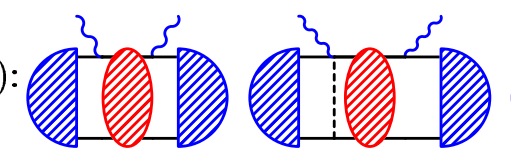

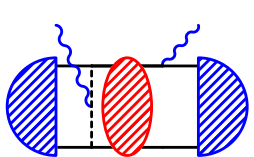

Figure 4: Diagrams that contribute to Compton scattering on the deuteron. The green blob in the one-body diagram (a) represents the graphs of Fig. 1, 2 while the red blob in (c) represents the full NN rescattering, including no interaction. The blue hemisphere represents the deuteron wave function. Figure reproduced from ref. [39]

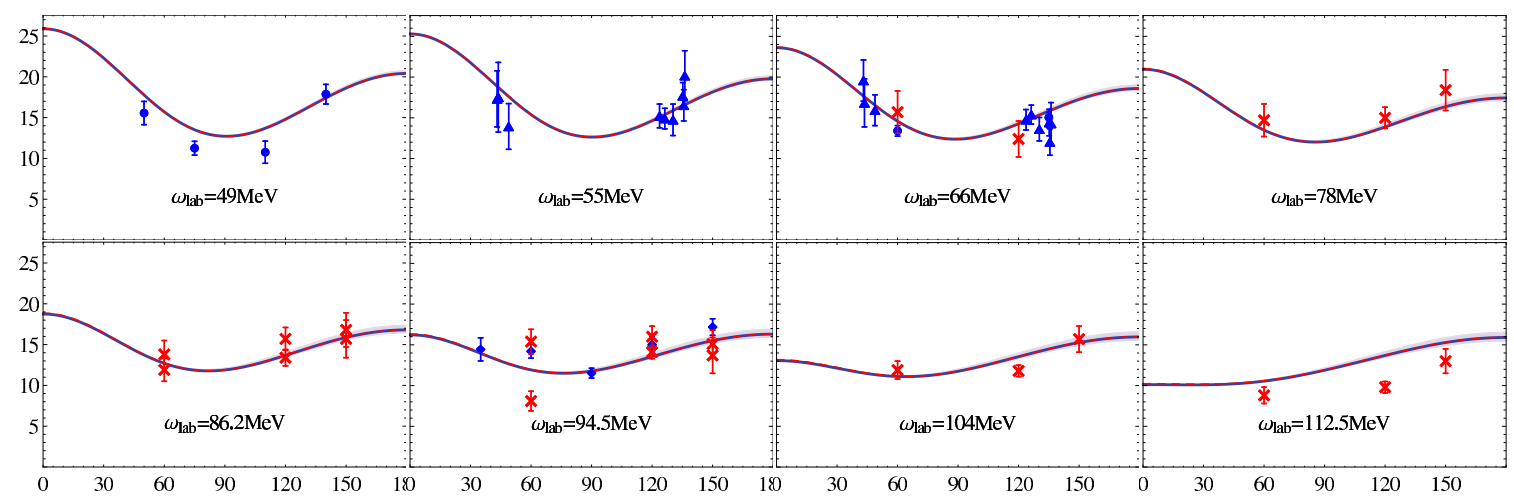

Figure 5: World deuteron Compton scattering data and the chiral EFT fit. The new data of Ref. [18] are the red crosses. Figure reproduced from ref. [18]

the new experiments at Mainz, the first of which is already published. We therefore look ahead to the prospects for confronting new data with the predictions of chiral EFT, and in particular for spin polarisabilities.

In Table 1 we show the predictions for the spin polarisabilities in two variants of $\chi$ EFT and two DR extractions, together with those published by Martel et al. [17]. Within error bars, we can see that there is a fair agreement between the various approaches, but there is certainly scope for experiment to pin down the values with greater accuracy.

\begin{tabular}{|l|l|l|l|rc|}
\hline & NNLO HB $\chi$ PT & NLO B $\chi$ PT & MAMI & DR(I) & DR(II) \\
\hline$\gamma_{E 1 E 1}^{\mathrm{p}}$ & $-1.1 \pm 1.9$ & $-3.3 \pm 0.8$ & $-3.5 \pm 1.2$ & -3.4 & -4.3 \\
$\gamma_{M 1 M 1}^{\mathrm{p}}$ & $2.2 \pm 0.5($ stat $) \pm 0.6$ & $2.9 \pm 1.5$ & $3.2 \pm 0.9$ & 2.7 & 2.9 \\
$\gamma_{E 1 M 2}^{\mathrm{p}}$ & $-0.4 \pm 0.6$ & $0.2 \pm 0.2$ & $-0.7 \pm 1.2$ & 0.3 & 0 \\
$\gamma_{M 1 E 2}^{\mathrm{p}}$ & $1.9 \pm 0.5$ & $1.1 \pm 0.3$ & $2.0 \pm 0.3$ & 1.9 & 2.2 \\
\hline$\gamma_{0}^{\mathrm{p}}$ & $-2.6 \pm 0.5($ stat $) \pm 1.8$ & $-0.9 \pm 1.4$ & $-1.0 \pm 0.1 \pm 0.1$ & -1.5 & -0.8 \\
$\gamma_{\pi}^{\mathrm{p}}$ & $5.5 \pm 0.5($ stat $) \pm 1.8$ & $7.2 \pm 1.7$ & $8.0 \pm 1.8$ & 7.8 & 9.4 \\
\hline
\end{tabular}

Table 1: Values of the proton spin polarisabilities from the current calculation [59], from covariant $\chi \mathrm{PT}$ at NLO [60], from experiment (multipoles: [17]; $\gamma_{0}:[61] ; \gamma_{\pi}:[62]$ ); and from Dispersion Relations (I) [41], (II) $[63,12,64]$ 

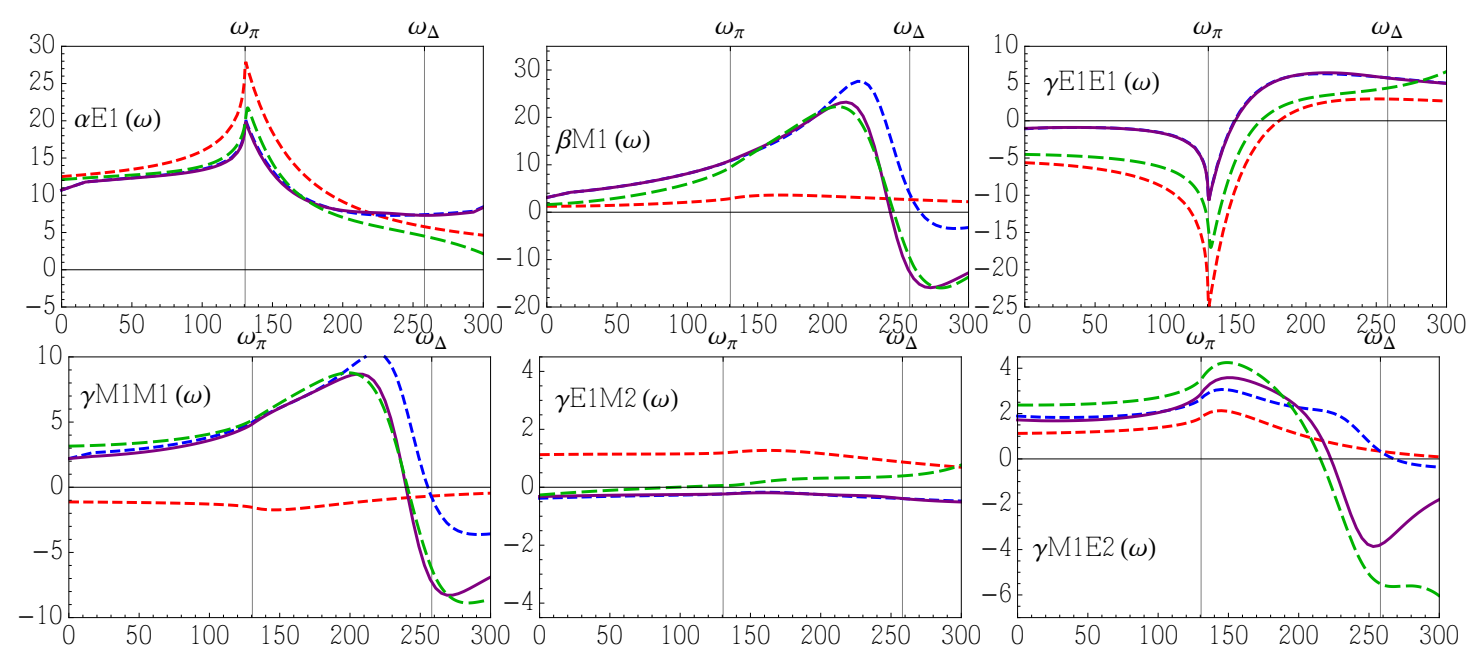

Figure 6: The six real parts of the $l=1$ multipoles as predicted by dispersion relations [12, 64] (green dashed) and by chiral EFT without the Delta (red dashed), with the Delta but without the $\gamma \mathrm{N} \Delta$ vertex loops of Fig, 2 (blue dashed) and with the vertex loops (purple solid). The horizontal axis is the centre-of-mass energy in $\mathrm{MeV}$, and the photoproduction cusp and Delta resonance peaks are marked with vertical lines.

A convenient way of encoding the low-energy Compton scattering amplitudes is via a multipole expansion [65, 12]. From appropriately-weighted angle integrals over the amplitudes, energydependent polarisabilities can be obtained, whose values as $\omega \rightarrow 0$ are just the usual (static) polarisabilities. At low energies the six $l=1$ multipoles are dominant, and even up to about $250 \mathrm{MeV}$, truncation at $l \leq 2$ gives a good approximation to the full cross section [12].

Figure 6 shows the six $l=1$ multipoles in chiral EFT. It is noticeable that the full predictions of chiral EFT (purple solid lines) and those of dispersion relations (green dashed lines) are very similar in shape at low energies, and their differences can mostly be reconciled though a shift in the $\omega=0$ values, that is, the static polarisabilities. It is interesting to see that in all the multipoles that are dominated by the Delta, the inclusion of $\gamma \mathrm{N} \Delta$ vertex loops substantially improves the agreement, which is qualitatively good even at the resonance. It would be very exciting to have enough cross-section and asymmetry data to extract the same quantities from experiment, but as yet that does not seem to be a realistic prospect. Still, the broad agreement of the shapes leads one to hope that fits to low-energy data using only the static polarisabilities as parameters ought to give rise to reasonably model-independent extractions.

For a spin- $\frac{1}{2}$ target, there are three asymmetries which are non-vanishing below the photoproduction threshold [41]. One, $\Sigma_{3}$, uses an unpolarised target and incoming photons polarised parallel or perpendicular to the reaction plane. The other two use a polarised target and right- and left-handed photons; for $\Sigma_{2 z}$ the target is polarised along the beam, whereas for $\Sigma_{2 x}$ it is perpendicular to the beam but still in the scattering plane. Preliminary low-energy data for $\Sigma_{3}$ from Mainz now exist [66]; $\chi$ EFT predictions for relevant energies are shown Fig. 7. It can clearly be seen that sensitivity to structure is present, but that high statistics will be needed to extract polarisabilities from this asymmetry alone. 

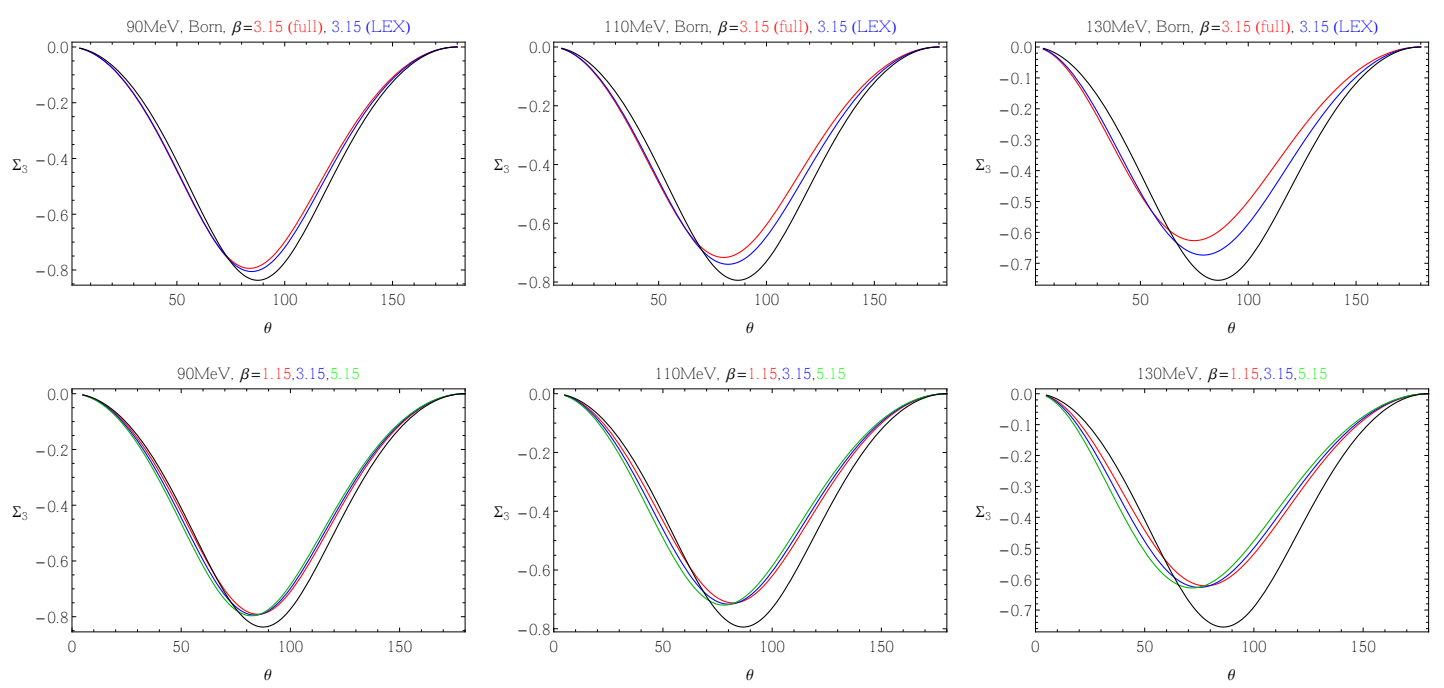

Figure 7: Predictions for the Mainz low-energy $\Sigma_{3}$ measurements. In the top panels the curves are Born (black), a low energy expansion (LEX) with structure entering only through only $\alpha_{E 1}^{\mathrm{p}}$ and $\beta_{M 1}^{\mathrm{p}}$ (blue) and the full $\chi$ EFT prediction. In the lower panel three different values of $\beta_{M 1}^{\mathrm{p}}$ are shown. $\alpha_{E 1}^{\mathrm{p}}=10.65$ is assumed throughout.

There is already published data from Mainz on $\Sigma_{2 x}$ and $\Sigma_{3}$ at higher energies [17, 67, 68]. These, together with dispersion-relation calculations [69], were used to produce the spin polarizability numbers quoted as "MAMI" in Table 1 . In Fig. 8. we show $\chi$ EFT curves compared to some of the data. The curves are not a fit, but only an exercise to explore the sensitivity to one of the spin polarisabilities, which is substantial. However this exercise comes with a significant caveat. The data is at (lab) energies of approaching $300 \mathrm{MeV}$. In this region, the $\chi$ EFT predictions are only NLO, and many other omitted diagrams would enter at lower orders than the LECs which govern spin polarisabilities. The $\gamma \mathrm{N} \Delta$ coupling constants are fit in this region, and hence are determined only to NLO; a 5\% increase of $g_{M}$ (bringing it close to the value assumed in Ref. [47]) will have as much effect as reducing $\gamma_{M 1 M 1}^{\mathrm{p}}$ by one unit.

\section{Future}

On the experimental front, publications from Mainz on $\Sigma_{3}$ for the proton at low and high energies are imminent, and $\Sigma_{2 z}$ should follow before too long. Further data taking on all of the asymmetries is proposed for the next two years. In addition experiments on unpolarised ${ }^{3} \mathrm{He}$ and on ${ }^{4} \mathrm{He}$ are scheduled for spring 2016, with a view to extracting the neutron scalar polarisabilities from a new target [70]. $\chi$ PT calculations (without the Delta) for ${ }^{3} \mathrm{He}$ already exist $[71,72]$, and these are being refined (eg by including the Delta) in preparation for the data analysis. More deuteron data from MAX-lab exists, and its analysis is ongoing [73]. HI $\gamma \mathrm{S}$ also has a Compton program, with data-taking on ${ }^{4} \mathrm{He}$ under way, deuteron runs planned for early 2016 and proton, both polarised and unpolarised, envisaged in the future [74]. 

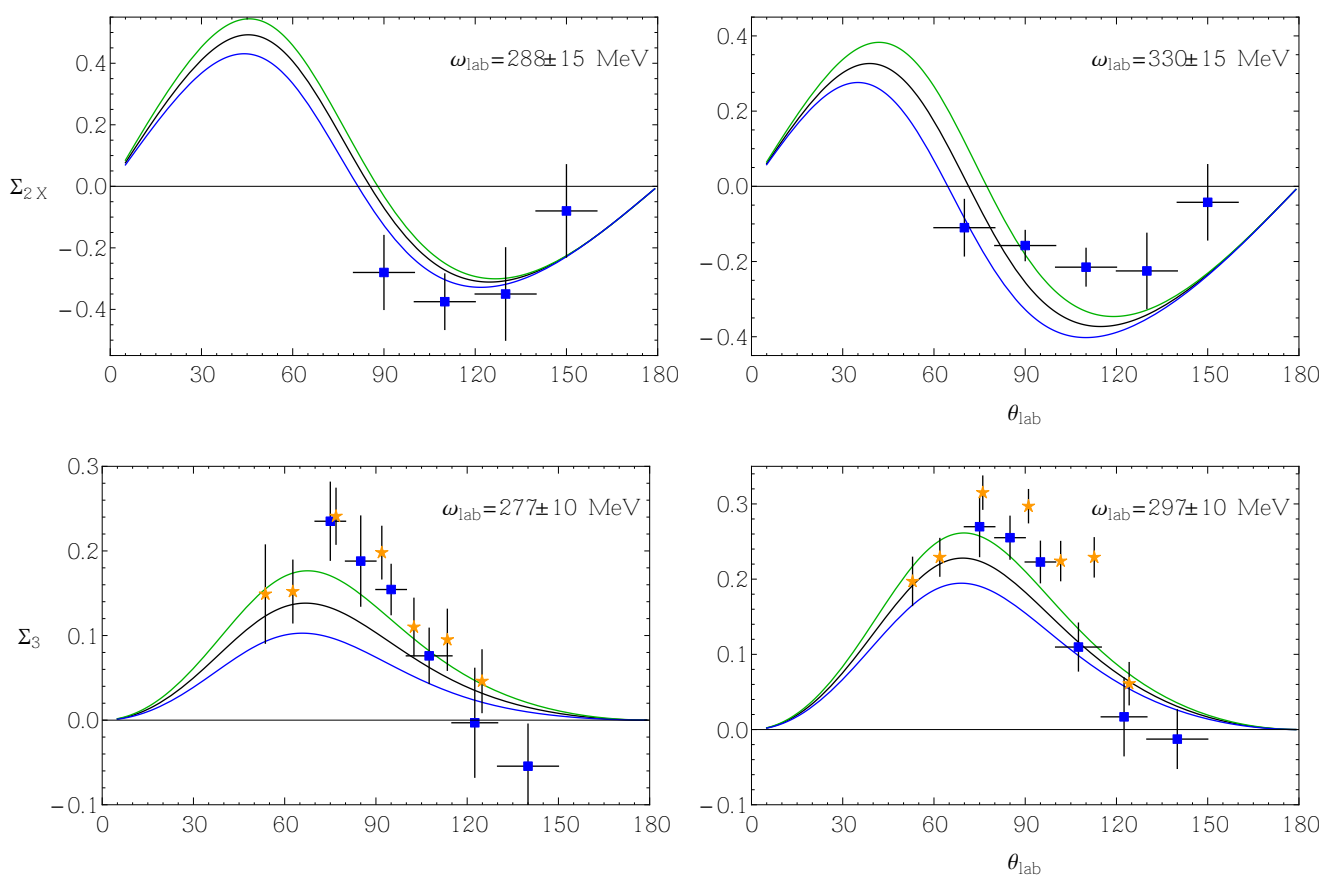

Figure 8: Published and unpublished data from Mainz (blue squares) and LEGS [48] (yellow stars), compared with prediction of $\chi \mathrm{EFT}$ with $\gamma_{M 1 M 1}^{\mathrm{p}}$ of 1.2 (green) 2.2 (black) and 3.2 (blue). Top: $\Sigma_{2 x}$, data from Refs [17] (left) and [67] (right). Bottom: $\Sigma_{3}$, Mainz data from Ref. [68].

On the theory side, work is in progress to publish the predictions of both heavy-baryon [49] and covariant [60] versions of $\chi$ EFT for proton and neutron spin polarisabilities (static and dynamic) and proton asymmetries. In Ref. [59, 49] the predictions - and their uncertainties-are extended to non-physical pion masses and compared with lattice data. And as already mentioned, work is in progress on an improved treatment of ${ }^{3} \mathrm{He}$.

By the time of CD2018, we should know considerably more about the polarisabilities of the proton and neutron.

\section{References}

[1] V. Bernard, N. Kaiser, J. Kambor and U. G. Meißner, Nucl. Phys. B 388 (1992) 315.

[2] V. Bernard, N. Kaiser, A. Schmidt and U. G. Meißner, Phys. Lett. B 319 (1993) 269 [arXiv:hep-ph/9309211].

[3] V. Bernard, N. Kaiser, U. G Meißner and A. Schmidt, Z. Phys. A 348 (1994) 317 [arXiv:hep-ph/9311354].

[4] V. Bernard, N. Kaiser and U. G. Meißner, Int. J. Mod. Phys. E 4 (1995) 193 [arXiv:hep-ph/9501384].

[5] X. -D. Ji, C. -W. Kao and J. Osborne, Phys. Rev. D 61 (2000) 074003 [arXiv:hep-ph/9908526].

[6] K. B. Vijaya Kumar, J. A. McGovern and M. C. Birse, arXiv:hep-ph/9909442.

[7] G. C. Gellas, T. R. Hemmert and U. -G. Meißner, Phys. Rev. Lett. 85 (2000) 14 [arXiv:nucl-th/0002027]. 
[8] K. B. Vijaya Kumar, J. A. McGovern and M. C. Birse, Phys. Lett. B 479 (2000) 167 [arXiv:hep-ph/0002133].

[9] J. A. McGovern, Phys. Rev. C 63 (2001) 064608 [Erratum-ibid. C 66 (2002) 039902] [nucl-th/0101057].

[10] S. R. Beane, M. Malheiro, J. A. McGovern, D. R. Phillips, U. van Kolck, Phys. Lett. B567 (2003) 200; Erratum-ibid. B607 (2005) 320. [arXiv:nucl-th/0209002].

[11] S. R. Beane, M. Malheiro, J. A. McGovern, D. R. Phillips and U. van Kolck, Nucl. Phys. A 747 (2005) 311 [arXiv:nucl-th/0403088].

[12] R. P. Hildebrandt, H. W. Grießhammer, T. R. Hemmert and B. Pasquini, Eur. Phys. J. A 20 (2004) 293 [arXiv:nucl-th/0307070].

[13] H. R. Weller, M. W. Ahmed, H. Gao, W. Tornow, Y. K. Wu, M. Gai and R. Miskimen, Prog. Part. Nucl. Phys. 62 (2009) 257.

[14] HI $\gamma$ S Programme-Advisory Committee Reports 2009 to 2014, with list of approved experiments at www.tunl.duke.edu/higs/experiments/approved/

[15] E. J. Downie and H. Fonvieille, Eur. Phys. J. ST 198 (2011) 287 [arXiv:1106.0232 [nucl-ex]].

[16] G. M. Huber and C. Collicott, arXiv:1508.07919 [nucl-ex].

[17] P. P. Martel et al. [A2 Collaboration], Phys. Rev. Lett. 114, no. 11, 112501 (2015) [arXiv:1408.1576 [nucl-ex]].

[18] L. S. Myers et al. [COMPTON@MAX-lab Collaboration], Phys. Rev. Lett. 113, no. 26, 262506 (2014) [arXiv:1409.3705 [nucl-ex]].

[19] L. Myers, J. Annand, J. Brudvik, G. Feldman, K. Fissum, H. Grießhammer, K. Hansen and S. Henshaw [COMPTON@MAX-lab Collaboration], Phys. Rev.C92 025203 (2015) [arXiv:1503.08094 [nucl-ex]].

[20] K. Pachucki, Phys. Rev. A 60 (1999) 3593.

[21] C. E. Carlson and M. Vanderhaeghen, arXiv:1109.3779 [physics.atom-ph].

[22] R. Pohl, R. Gilman, G. A. Miller and K. Pachucki, Ann. Rev. Nucl. Part. Sci. 63, 175 (2013) [arXiv:1301.0905 [physics.atom-ph]].

[23] M. C. Birse and J. A. McGovern, Eur. Phys. J. A 48 (2012) 120 [arXiv:1206.3030 [hep-ph]].

[24] A. Walker-Loud, C. E. Carlson and G. A. Miller, PoS LATTICE 2012, 136 (2012) [arXiv:1210.7777 [hep-lat]].

[25] A. Walker-Loud, C. E. Carlson and G. A. Miller, Phys. Rev. Lett. 108 (2012) 232301 [arXiv:1203.0254 [nucl-th]].

[26] F. B. Erben, P. E. Shanahan, A. W. Thomas and R. D. Young, Phys. Rev. C 90, no. 6, 065205 (2014) [arXiv:1408.6628 [nucl-th]].

[27] A. W. Thomas, X. G. Wang and R. D. Young, Phys. Rev. C 91, no. 1, 015209 (2015) [arXiv:1406.4579 [nucl-th]].

[28] J. Gasser, M. Hoferichter, H. Leutwyler and A. Rusetsky, arXiv:1506.06747 [hep-ph].

[29] E. Chang, W. Detmold, K. Orginos, A. Parreno, M. J. Savage, B. C. Tiburzi and S. R. Beane, arXiv:1506.05518 [hep-lat]. 
[30] M. Lujan, A. Alexandru, W. Freeman and F. Lee, PoS LATTICE 2014, 153 (2014) [arXiv:1411.0047 [hep-lat]].

[31] W. Detmold, B. C. Tiburzi and A. Walker-Loud, Phys. Rev. D 81, 054502 (2010) [arXiv:1001.1131 [hep-lat]].

[32] T. Primer, W. Kamleh, D. Leinweber and M. Burkardt, Phys. Rev. D 89, no. 3, 034508 (2014) [arXiv:1307.1509 [hep-lat]].

[33] J. M. M. Hall, D. B. Leinweber and R. D. Young, Phys. Rev. D 89, no. 5, 054511 (2014) [arXiv:1312.5781 [hep-lat]].

[34] M. Engelhardt, PoS LATTICE 2011, 153 (2011) [arXiv:1111.3686 [hep-lat]].

[35] M. Engelhardt [LHPC Collaboration], Phys. Rev. D 76, 114502 (2007) [arXiv:0706.3919 [hep-lat]].

[36] M. Engelhardt, PoS LAT 2009, 128 (2009) [arXiv:1001.5044 [hep-lat]].

[37] M. Engelhardt, J. Saenz, R. Höllwieser, private communication and forthcoming.

[38] W. Freeman, A. Alexandru, M. Lujan and F. X. Lee, Phys. Rev. D 90, no. 5, 054507 (2014) [arXiv:1407.2687 [hep-lat]].

[39] H. W. Grießhammer, J. A. McGovern, D. R. Phillips and G. Feldman, Prog. Part. Nucl. Phys. 67 (2012) 841. [arXiv:1203.6834 [nucl-th]].

[40] J. A. McGovern, D. R. Phillips and H. W. Grießhammer, Eur. Phys. J. A 49 (2013) 12 [arXiv:1210.4104 [nucl-th]].

[41] D. Babusci, G. Giordano, A. I. L’vov, G. Matone and A. M. Nathan, Phys. Rev. C 58 (1998) 1013. [arXiv:hep-ph/9803347].

[42] E. E. Jenkins, A. V. Manohar, In Dobogokoe 1991, Proceedings, Effective field theories of the standard model 113-137 and Calif. Univ. San Diego - UCSD-PTH 91-30 (91/10,rec.Dec.) 26 p. (201392) (see Conference Index).

[43] T. R. Hemmert, B. R. Holstein and J. Kambor, Phys. Rev. D 55 (1997) 5598 [arXiv:hep-ph/9612374].

[44] T. R. Hemmert, B. R. Holstein, J. Kambor and G. Knochlein, Phys. Rev. D 57 (1998) 5746 [arXiv:nucl-th/9709063].

[45] V. Pascalutsa, D. R. Phillips, Phys. Rev. C67 (2003) 055202. [nucl-th/0212024].

[46] V. Pascalutsa and M. Vanderhaeghen, Phys. Rev. D 73 (2006) 034003 [arXiv:hep-ph/0512244].

[47] V. Pascalutsa, M. Vanderhaeghen and S. N. Yang, Phys. Rept. 437 (2007) 125 [arXiv:hep-ph/0609004].

[48] G. Blanpied et al., Phys. Rev. C 64 (2001) 025203

[49] H. W. Grießhammer, these proceedings.

[50] E. L. Hallin et al., Phys. Rev. C 48 (1993) 1497.

[51] S. Wolf et al., Eur. Phys. J. A 12 (2001) 231.

[52] V. Olmos de León et al., Eur. Phys. J. A 10 (2001) 207.

[53] V. Lensky and V. Pascalutsa, Pisma Zh. Eksp. Teor. Fiz. 89 (2009) 127 [JETP Lett. 89 (2009) 108] [arXiv:0803.4115 [nucl-th]].

[54] V. Lensky, V. Pascalutsa, Eur. Phys. J. C65 (2010) 195-209. [arXiv:0907.0451 [hep-ph]]. 
[55] V. Lensky, J. A. McGovern, D. R. Phillips and V. Pascalutsa, Phys. Rev. C 86, 048201 (2012) [arXiv:1208.4559 [nucl-th]].

[56] V. Lensky and J. A. McGovern, Phys. Rev. C 89 (2014) 3, 032202 [arXiv:1401.3320 [nucl-th]].

[57] R. P. Hildebrandt, H. W. Grießhammer and T. R. Hemmert, Eur. Phys. J. A 46 (2010) 111 [arXiv:nucl-th/0512063].

[58] M. I. Levchuk and A. I. L’vov, Nucl. Phys. A 674 (2000) 449. [arXiv:nucl-th/9909066].

[59] H. W. Grießhammer, J. A. McGovern and D. R. Phillips, forthcoming.

[60] V. Lensky, J. McGovern and V. Pascalutsa, arXiv:1510.02794 [hep-ph].

[61] H. Dutz, K. Helbing, J. Krimmer, T. Speckner and G. Zeitler [GDH and A2 Collaborations], Phys. Rev. Lett. (2003) 91.

[62] M. Camen et al., Phys. Rev. C 65 (2002) 032202.

[63] B. R. Holstein, D. Drechsel, B. Pasquini and M. Vanderhaeghen, Phys. Rev. C 61 (2000) 034316 [arXiv:hep-ph/9910427].

[64] B. Pasquini, private communication based on Ref. [12].

[65] H. W. Grießhammer and T. R. Hemmert, Phys. Rev. C 65 (2002) 045207 [arXiv:nucl-th/0110006].

[66] V. Sokhoyan and E. Downie, private communication, paper in preparation.

[67] P. P. Martel, PhD thesis: U. Mass. Amherst, 2013.

[68] C. Collicott, PhD thesis: Dalhousie, 2015.

[69] D. Drechsel, B. Pasquini and M. Vanderhaeghen, Phys. Rept. 378 (2003) 99 [arXiv:hep-ph/0212124].

[70] J. Annand, these proceedings

[71] D. Choudhury, A. Nogga and D. R. Phillips, Phys. Rev. Lett. 98 (2007) 232303 [arXiv:nucl-th/0701078].

[72] D. Shukla, A. Nogga and D. R. Phillips, Nucl. Phys. A 819 (2009) 98 [arXiv:0812.0138 [nucl-th]].

[73] G. Feldman, these proceedings.

[74] H. Gao, these proceedings. 